\title{
Impacto de la implementación de un modelo ecoformativo a través de proyectos estratégicos: el caso de la Universidad Castro Carazo (Costa Rica)
}

Impact of the Implementation of an Eco-formative Model through Strategic Projects: the Experience of Castro Carazo University (Costa Rica)

Impacte de la implementació d'un model ecoformatiu a través de projectes estratègics: el cas de la Universidad Castro Carazo (Costa Rica)

\author{
Stefany Ocampo-Hernández ${ }^{1, *(\odot)}$, Luis Alonso Arrieta-Ávila ${ }^{2 \oplus}$, Virginia \\ Aguilar-Barquero ${ }^{30}$
}

1 | Innovación Curricular, Universidad Castro Carazo, San José, Costa Rica
2 | Calidad Académica, Universidad Castro Carazo, San José, Costa Rica
3 | Laboratorio de Aprendizaje, Universidad Castro Carazo, San José, Costa Rica
${ }^{*}$ Autor para correspondencia: socampo@castrocarazo.ac.cr (Stefany Ocampo-Hernández)

Recibido: 09/10/2019 | Aceptado: 11/03/2020 | Publicado: 24/06/2020

Cómo citar:

Ocampo-Hernández, S., Arrieta-Ávila, L.A., y Aguilar-Barquero, V. (2020). Impacto de la implementación de un modelo ecoformativo a través de proyectos estratégicos: el caso de la Universidad Castro Carazo (Costa Rica). Research in Education and Learning Innovation Archives, 24,56-71.

10.7203/realia.24.15898

Copyright: El/La Autor/a. Open Access: Este es un artículo de acceso abierto distribuido bajo los términos de la licencia Creative Commons

Attribution-NoDerivatives 4.0 International licence (CC BY-ND 4.0)

Financiación: None informed
RESUMEN: La Universidad Castro Carazo ha adoptado un modelo educativo de ecoformación desde el año 2016. Para esto, ha sido necesaria la incorporación de buenas prácticas y estrategias de innovación pedagógica y curricular, como parte integral de sus procesos educativos y administrativos. En este artículo se detallan las prácticas y estrategias más relevantes: el diseño e implementación de un programa de especialización en docencia universitaria, la inauguración de un laboratorio de aprendizaje para el fomento del aprendizaje mediante la fabricación digital, los multientornos, y los mapas conceptuales, así como el fortalecimiento de un equipo de innovación curricular. Estos procesos disruptivos han cambiado la dinámica docentes-discentes, al promover un diálogo de saberes a través de espacios físicos y virtuales, donde el error es parte del aprendizaje, y donde la ecología de saberes se vuelve la base del conocimiento.

PALABRAS CLAVE: ecoformación; aprendizaje; docencia; fabricación digital; mapas conceptuales; entorno virtual; innovación curricular

ABSTRACT: Castro Carazo University has adopted an educational model of ecoformation since 2016. It has been necessary to incorporate good practices and strategies of pedagogical and curricular innovation, as an integral part of its educational and administrative processes. This article details the most relevant practices and strategies: the design and implementation of a university teaching program for faculty, the inauguration of a learning laboratory for the promotion of learning through digital fabrication, and the strengthening of a Curricular Innovation team. These disruptive processes have changed the teacher-student dynamics, by promoting a dialogue of knowledge through physical and virtual spaces, where error is part of learning, and where the ecology of knowledge becomes the basis of learning.

KEYWORDS: ecoformation; learning; facilitating learning; digital fabrication; concept maps; virtual learning environments; curricular innovation 
RESUM: La Universitat Castro ha adoptat un model educatiu des de l'any 2016. Per a això, ha estat necessària la incorporació de bones pràctiques i estratègies d'innovació pedagògica i curricular com a part integral dels seus processos educatius i administratius. En aquest article es detallen les pràctiques i estratègies més rellevants; per exemple: el disseny i implementació d'un programa d'especialització en docència universitària, la inauguració d'un laboratori d'aprenentatge per al foment de l'aprenentatge mitjançant la fabricació digital, l'ús de mapes conceptuals com a eina per a representar el coneixement, i l'enfortiment d'un equip d'innovació curricular. Aquests processos disruptius han canviat la dinàmica docents-discents, perquè promouen un diàleg de sabers a través d'espais físics i virtuals, en què l'error és part de l'aprenentatge, i en què l'ecologia de sabers es torna la base del coneixement.

PARAULES CLAU: ecoformació; aprenentatge; docència; fabricació digital; mapes conceptuals; entorn virtual; innnovació curricular

Notas de aplicación práctica

\section{Qué se sabe sobre el tema}

- En su artículo "La crisis planetaria y la ecoformación", Badilla-Saxe (2018a) explica que los sistemas educativos deben ser capaces de desarrollar la consciencia indispensable para resolver problemas planetarios, de visibilizar las interrelaciones e interdependencias entre el mundo social, cultural, personal y natural, el cual debe incorporar el uso de didácticas innovadoras y tecnologías de punta, pero que debe trascender a una consciencia planetaria. De esta manera, la autora invita a replantear la universidad desde una visión integral y compleja para visibilizar los aprendizajes que se generan dentro y fuera de las aulas y que corresponden no solo a los contenidos de los cursos, sino a la formación integral de una ciudadanía crítica y consciente, capaz de generar transformaciones sociales a su alrededor.

\section{Qué aporta este trabajo}

- Muestra la ruta de aprendizaje de la Universidad Castro Carazo desde el 2016 cuando adopta el modelo educativo de ecoformación como parte integral de sus procesos y proyectos estratégicos, acciones didácticas y estrategias de innovación pedagógica y curricular. Como parte de esta evolución educativa, la universidad ha incorporado una serie buenas prácticas y de estrategias educativas innovadoras, que pueden servir de inspiración y referencia para otras organizaciones educativas o instituciones de educación superior que también buscan atender las crisis ya antes mencionadas y optar por un Paradigma Emergente.

\section{Implicaciones para la práctica y / o política}

- Se comparten experiencias exitosas y lecciones aprendidas que pueden servir de referencia para delimitar una estrategia efectiva de proyectos para la promoción de innovación en el aprendizaje dentro de instituciones de educación superior.

\section{INTRODUCCIÓN}

\subsection{Historia de la Universidad Castro Carazo}

Desde su fundación en 1936, la Universidad Castro Carazo se ha esforzado por brindar oportunidades de formación en la educación superior, inspiradas en el pensamiento humanista de su fundador, Miguel Ángel Castro Carazo. Partiendo de este pensamiento, la Universidad se ha transformado en una comunidad de a aprendizaje para el desarrollo pleno de las facultades intelectuales, emocionales, físicas y profesionales de sus estudiantes, con el fin de que estos participen de manera solidaria en el ejercicio de su ciudadanía y eficazmente en su desempeño laboral. 


\subsection{Modelo Educativo}

En ese contexto, el modelo educativo de la Universidad Castro Carazo responde a su misión, visión, valores, compromiso ecológico y perfil general de la persona graduada. Refleja la mirada del paradigma emergente, inclusivo y holista que propone el desarrollo del pensamiento complejo para poder enfrentar de manera solidaria y con una ética planetaria las realidades del mundo actual. Se fundamenta en una pedagogía constructivista social y ecoformativa que, en una espiral recursiva, enlaza permanentemente teoría y práctica; lleva en su seno el germen de la actualización permanente e incluye una mediación docente activa y respetuosa, que busca apoyar al estudiantado en su aprendizaje mediante acciones didácticas colaborativas.

La Dra. Eleonora Badilla Saxe ${ }^{1}$ actual rectora de la institución, continúa impulsando los esfuerzos de nuestro fundador y con esta misión, es que se realizan mejoras que buscan la interrelación del individuo con su contexto biológico y social, el modelo denominado ecoformativo, definido por Acuña como:

Aprender sobre el mundo actual, el contexto que nos rodea y los fenómenos contemporáneos globales (la realidad que se viven alrededor del planeta). Teniendo presente las interacciones individuo-sociedadnaturaleza; como parte de eso, enfatizando en el crecimiento personal, pero siempre en armonía con el planeta y todos sus seres vivientes

(2017, p. 17)

La Universidad se encuentra en un proceso de mejora continua con una visión ecosistémica para la cual define como necesario innovar y diversificar las experiencias de aprendizaje en el marco de su plan estratégico quinquenal 2018-2022, el cual busca atender las necesidades del alumnado, el personal académico administrativo, docente y la realidad nacional. Dichas necesidades, se consideran a través de una serie de proyectos estratégicos desde las instancias de gobernanza:

- Programa universitario especializado en docencia universitaria (PUEDO)

- Laboratorio de Aprendizaje (A:Lab), el cual promueve el uso de fabricación digital, Entornos Virtuales de aprendizaje y de mapas conceptuales.

- Área de Innovación Curricular

Asimismo, el modelo educativo se fundamenta en los siguientes pilares que sustentan cada uno de los proyectos estratégicos mencionados (Badilla-Saxe, 2018b):

- El paradigma emergente: Se considera prioritario cambiar el paradigma tradicional con que se veía el mundo antes del siglo XX y comprender que hay un nuevo paradigma que emerge. Es un paradigma holista, solidario, con un gran compromiso por el equilibrio de la vida en el planeta. Se trata de una evolución de la fragmentación en la lógica cartesiana y newtoniana (que la incluye, pero la trasciende) que pretende religar, reunir y asociar lo que ha sido artificialmente separado (Badilla-Saxe, 2005). Es un paradigma que, al decir de Henao-Willis (1999) quiere superar las díadas, las relaciones elementales de oposición y polarización excluyente (sujeto-objeto; alma-cuerpo; espíritu-materia; calidad-cantidad; finalidadcausalidad; sentimiento-razón; existencia esencia) para dar paso a una mirada más incluyente, holista, ecosistémica y compleja.

- El pensamiento complejo: Para comprender el paradigma emergente y cambiar la forma de ver el mundo se hace imprescindible que la forma de pensar deje de ser simplificada y lineal y evolucione hacia un pensamiento complejo, ecológico, en 
virtud de que, como dice Moraes (2010), existe una interdependencia ecosistémica entre los seres humanos, el medio ambiente y el pensamiento; entre los seres humanos y sus procesos de desarrollo, entre el sujeto y el contexto, entre quien educa y quien se educa; entre el sujeto y el objeto: entre el ser, conocer, hacer y el vivir-convivir, entre la ontología y la epistemología, que conecta el ser, el saber y hacer.

- La ética planetaria y el compromiso ecológico: Es necesario reconocer el papel fundamental de una educación transformadora que se centre en la condición humana, en el desarrollo de la comprensión, la sensibilidad y la ética, en la diversidad cultural y la pluralidad de individuos; una educación que privilegie la construcción de un conocimiento pertinente, de naturaleza transdisciplinar, tomando en cuenta siempre las relaciones entre el individuo, la sociedad y la naturaleza (Moraes, 2010)

- Constructivismo y el aporte de las Neurociencias: Tal como afirma Castro (2009), los estudios en neurociencia han venido a confirmar lo que muchos investigadores han sospechado: las personas activamente construyen sus propias representaciones de una realidad objetiva utilizando su conocimiento previo. Afirma la autora que, al no compartir las mismas experiencias de vida, cada persona tiene una interpretación y un proceso de construcción del conocimiento distinto. Esta visión del aprendizaje se conoce como constructivismo, cuyos exponentes más importantes fueron John Dewey (1859-1952), Jean Piaget ( 1896-1980), Jerome Bruner (1915) y Lev Vygotsky (1896-1934).

\section{MATERIALES Y METODOLOGÍA}

Desde un enfoque cualitativo, la sistematización de experiencias es una de las herramientas metodológicas que está referida, necesariamente, a experiencias prácticas concretas, Jara la define como:

Un proceso permanente, acumulativo, de creación de conocimientos a partir de nuestra experiencia de intervención en una realidad social, como un primer nivel de teorización sobre la práctica. En este sentido, la sistematización representa una articulación entre teoría y práctica... y sirve a objetivos de los dos campos. Por un lado, apunta a mejorar la práctica, la intervención, desde lo que ella misma nos enseña...; de otra parte..., aspira a enriquecer, confrontar y modificar el conocimiento teórico actualmente existente, contribuyendo a convertirlo en una herramienta realmente útil para entender y transfo rmar nuestra propia realidad

(1994, p. 20).

Dicha articulación entre teoría y práctica se vislumbra a continuación, al mostrar el impacto de la implementación del modelo ecoformativo a través de tres proyectos estratégico. La sistematización de estos proyectos busca brindar una interpretación crítica de los mismos (Barnechea-García y Morgan-Tirado, 2010), a partir de su ordenamiento y reconstrucción, para confrontar estas experiencias con otras y con el conocimiento teórico existente, contribuyendo a la generación de conocimiento y lecciones aprendidas útiles a otras instituciones de educación superior.

Siendo este el propósito del presente documento, es que se comparte la ruta de aprendizaje de la universidad en la vivencia de su modelo educativo desde la experiencia de los sujetos participantes, mediante la reconstrucción histórica de un proceso vivo de lo acontecido (Picado-Alfaro, 2018). Continuando con el editorial publicado por la revista Magisterio, la elaboración de la reconstrucción permite: 
Mostrar la experiencia de los sujetos de la práctica, a construir el nudo relacional desde las acciones que la constituyen, que se forjan en la interacción del sujeto con el mundo, que hace que estos sean complejos, reflexivos, innovadores, empíricos, pero ante todo, cuando se colocan las posibilidades para realizarlo, productores de saber y de teoría

(Magisterio, 2008 , p. 6).

\subsection{Programa universitario especializado en docencia (PUEDO )}

El cambio de modelo educativo trajo consigo la oportunidad de brindar al cuerpo docente las herramientas y estrategias necesarias para generar una educación disruptiva, que trascienda la visión tradicional del docente como eje central de la enseñanza, y donde se propicie el desarrollo de espacios de aprendizaje desde las y los discentes. Para esto, se crea un programa de especialización en docencia universitaria (PUEDO), de modo que las y los docentes pueden experimentar el modelo de ecoformación de primera mano y desarrollar los conocimientos y habilidades para aplicarlo.

Este programa de especialización está compuesto por las siguientes dimensiones (ver Figura 1):

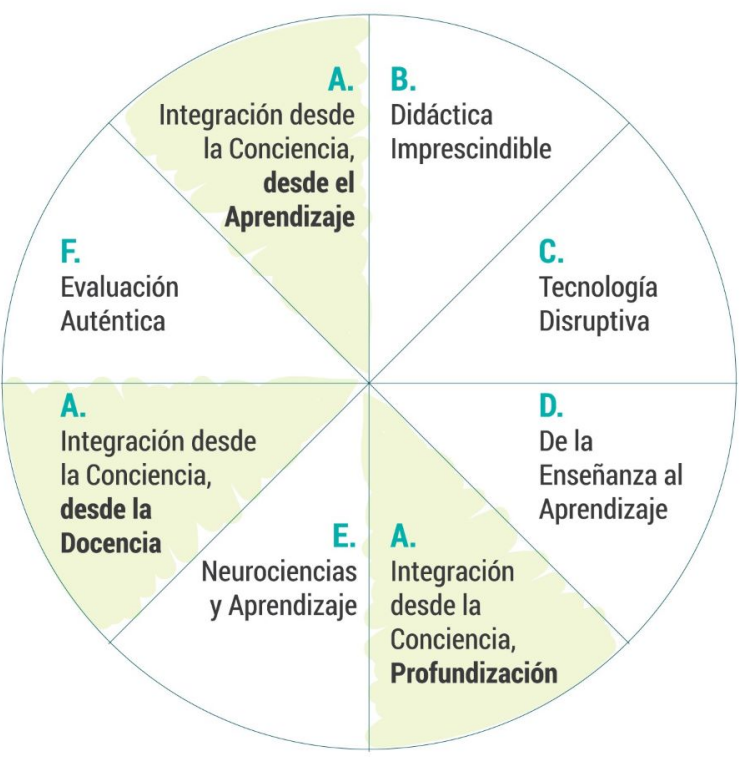

Figura 1. Dimensiones del programa especializado en docencia universitaria. Fuente: elaboración propia.

Como se observa en la Figura 1, el PUEDO tiene una estructura circular, recursiva, donde las personas participantes aprenden desde la práctica hacia la teoría, y realizan pausas estratégicas para la conciencia y auto-percepción sobre el proceso de aprendizaje que viven a lo largo del programa, así como sobre sus prácticas docentes y sus concepciones previas del aprendizaje universitario dimensión A: "Integración desde la Conciencia").

Durante la dimensión B: "Didáctica Imprescindible", se busca reflexionar sobre el rol del docente en el proceso educativo y la integración de enfoques y estrategias didácticas centradas en el alumnado (e.g.: pensamiento visible, clase inversa, aprendizaje basado en proyectos, enseñanza para la comprensión, ludificación). Mientras que en "Tecnología Disruptiva" (C) se desarrollan herramientas para la mediación docente 
en entornos digitales y el uso de herramientas tecnológicas de manera disruptiva, evitando reproducir patrones de educación tradicional.

El modelo de ecoformación y el cambio de paradigma que se crea a partir de la "Escuela Nueva", se viven a través de la dimensión D: "De la enseñanza al aprendizaje", donde se enfatiza la necesidad de hacer a las y los estudiantes el centro activo, reflexivo y participativo de los procesos de construcción del aprendizaje. "Neurociencias y aprendizaje" (E), busca desarrollar un aprendizaje real, entendiendo cómo funciona el cerebro humano y cuáles son los aportes actuales de las neurociencias a la educación. Finalmente, durante "Evaluación Auténtica" (F), se brinda coherencia entre los procesos de aprendizaje y la evaluación de los mismos, entendiendo que una evaluación auténtica parte de las experiencias, la práctica y la integración de los conocimientos y no de un proceso memorístico y/o punitivo.

A la fecha, 84 docentes han participado en PUEDO, lo que representa un $38 \%$ del total de docentes de la universidad, lo que refleja los esfuerzos por implementar el modelo de ecoformación.

Como parte de la segunda generación del PUEDO, durante el módulo de "Didáctica Imprescindible", se consultó a 39 docentes sobre su percepción inicial del rol docente dentro de los procesos de aprendizaje, mediante la recolección de videos con las opiniones de las personas participantes al momento de iniciar el proceso y en la última sesión del mismo, de manera que se puedan comparar las respuestas y analizar las ideas y las creencias manifestadas en sus discursos.

Se seleccionó esta estrategia dado que plantea una perspectiva nueva para analizar la conciencia sobre un tema determinado, tal como menciona Adamini cuando indica que:

El análisis del discurso es señalado como una perspectiva teóricametodológica que aborda a los discursos como espacios de formación subjetiva, analizando la incidencia del sujeto (enunciador/locutor) y su contexto (de enunciación/producción/recepción). Esto implica que comprende a las narraciones como un espacio simbólico en donde los sujetos se posicionan subjetivamente, dejando marcas de sí y de su contexto. En función a ello, es considerada una perspectiva analítica pertinente para adentrarse en el complejo e inestable mundo de las identificaciones de los sujetos.

(2016, p. 2)

De esta manera, se buscó valorar si el PUEDO generó algún cambio en la mentalidad y la actitud de las y los participantes, a través del análisis de las ideas y creencias sobre el rol docente manifestadas por las personas participantes durante la primera y la última sesión del módulo de" Didáctica Imprescindible" del programa.

\subsection{Laboratorio de aprendizaje (A: Lab)}

El tránsito hacia un nuevo paradigma incluye una re-conceptualización de los entornos y/o multientornos donde se aprende haciendo y fabricando. En septiembre del 2017, se inauguró el Laboratorio de Aprendizaje (A:Lab), como un ambiente de aprendizaje flexible, que permite diversas configuraciones del espacio, con mobiliario alternativo y tecnologías para la creación y la fabricación digital. El énfasis de este espacio no es la fabricación de prototipos (aunque se fabrican) ni las tecnologías digitales (aunque se utilizan), sino el aprendizaje (Badilla-Saxe et al., 2018).

$\mathrm{Su}$ objetivo ha sido el de ofrecer a la comunidad educativa un espacio innovador, atractivo y adecuado para la exploración de proyectos inter y transdisciplinarios, mediante el aprendizaje activo (aprender/haciendo, aprender/fabricando) que promueva el pensamiento complejo, la conciencia planetaria y habilidades para la vida, como: 
colaboración, comunicación, solución de problemas, pensamiento crítico y creatividad. Su diseño e implementación se fundamenta en teorías como el Constructivismo, el Constructivismo social y el Construccionismo. En particular, se parte del Construccionismo como una visión educativa innovadora sobre el uso de las tecnologías digitales para apoyar el aprendizaje de las personas que, además, permite comprender la forma en que la tecnología digital es apropiada por la sociedad y por el individuo (Papert, 1990).

El A:Lab se inspira en la tendencia mundial de fabricación digital que se promueve en los "Espacios Maker" o "Fab Labs", pero se enfoca en el aprendizaje y procesos de construcción de conocimiento que pueden ser impulsados y potenciados por medio de experiencias colaborativas de fabricación con tecnologías digitales. Desde la construcción de mapas conceptuales sobre temas complejos, hasta la creación de prototipos y soluciones creativas a través de la computación física o la impresión 3D. Por lo tanto, el A:Lab busca trascender el activismo (en el que incurre la mayoría de "Maker spaces"), para hacer visibles los procesos de pensamiento profundo y aprendizaje que pueden promoverse a través de la fabricación digital.

Para alcanzar este objetivo, el A:Lab, a través de un grupo interdisciplinar de profesionales, incuba y pone a prueba nuevas formas de usar la fabricación digital para promover un aprendizaje significativo en cada una de las especialidades que ofrece la universidad. Ello implica indagación constante sobre nuevas herramientas y metodologías, así como la articulación y comunicación constante con docentes y encargados de cátedra, para asegurar la pertinencia de cada experiencia según los planes curriculares y objetivos educativos a fortalecer. De esta manera, las experiencias dentro del A:Lab amplían las oportunidades de aprendizaje del estudiantado, a la vez que se convierten en modelos de maneras prácticas de la integración efectiva de las tecnologías digitales y la fabricación en los procesos de aprendizaje que ocurren en todas las disciplinas de la universidad. Con esto se espera un efecto exponencial, donde las experiencias (modelos) trascienden el laboratorio para ser replicadas o adaptadas por cada docente en sus aulas regulares, según el contexto y las necesidades educativas particulares.

\subsubsection{Mapas conceptuales}

Según Freire (2002) un cambio de paradigma requiere de innovación y de la modificación del quehacer; con innovación no se rechaza ni se discrimina lo que se hace, al contrario, se canaliza de otras formas. En esta línea por visibilizar el modelo, es que se comienza a "Aprender a Mapear" en el 2016, acompañados por la mirada experta del Dr. Alberto Cañas Collado ${ }^{2}$ y el apoyo pedagógico de la Dra. Eleonora Badilla Saxe en un taller de 60 horas. Durante este proceso surge la primera generación de Maperos, una comunidad de aprendizaje conformada por 25 aprendientes, entre ellos docentes, directores académicos y administrativos.

La teoría de Mapas Conceptuales (MC) fue desarrollada en la década de 1970 por el docente e investigador de la Universidad de Cornell, Joseph Donald Novak. Para J. Novak (2010), al aprender por repetición, no se hace ningún esfuerzo para relacionar nuevas ideas con los conocimientos previos que sean relevantes en la estructura cognitiva. No obstante, en el aprendizaje significativo, el aprendiente elige integrar de manera sustantiva nuevos conceptos y proposiciones con ideas relevantes existentes en su estructura cognitiva.

Precisamente por lo anterior, la universidad reconoce a los MC como herramientas poderosas del pensamiento, al hacer énfasis en los conocimientos previos del estudiante y el error como oportunidad de aprendizaje (J. D. Novak y Gowin, 1984). Para J. Novak (2010), es durante la construcción de los mapas conceptuales que se puede tener una concepción equivocada, es decir, una interpretación no aceptada (no necesariamente errónea) de una proposición; este "error" concede la posibilidad de 
negociar el significado sobre esa concepción, de ahí su importancia al convertirse en una oportunidad de aprendizaje. Al revelar los MC la estructura cognitiva de los aprendientes, los docentes tienen la oportunidad de notar el nivel de comprensión de sus estudiantes, incluso cuando se cometen errores conceptuales. Por el contrario, si enjuician el error, se eliminan las oportunidades de mejora y la actitud hacia el aprendizaje se puede ver afectada.

En esta línea sobre el uso de los MC en la práctica docente, una estrategia didáctica ideal para trabajar con MC es el aprendizaje colaborativo. Según Collazos, Guerrero, y Vergara (2002), a quienes cita Salas-Alvarado (2016) "es básicamente el uso de grupos de tal manera que el estudiantado, mediante el trabajo conjunto, logre maximizar su aprendizaje y el de los demás individuos, la idea es lograr un conjunto que trabaja para la totalidad de miembros en busca de un fin común que engrandezca el proceso de aprendizaje" (Salas-Alvarado, 2016, p. 6). Esta estrategia requiere de los conocimientos previos y del acompañamiento, siendo elegida como parte de la metodología de los facilitadores a cargo del PUEDO para apoyar a los docentes.

En palabras de Cañas, Reiska, y Möllits (2017) es posiblemente más importante el proceso que el mapa final, sobre todo porque el interés principal es aprender, ejercitar habilidades de pensamiento de orden superior y generar un cuestionamiento continuo entre el estudiante y el educador. De esta manera, un buen MC contiene proposiciones relevantes en su totalidad y explica ampliamente la pregunta de enfoque, mas no debería ser descriptivo, sino explicativo. Este es uno de los aspectos que resulta ser complejo de entender para los docentes; primero, diferenciar un MC de un esquema y segundo, ver el ejercicio de equivocarse como un aprendizaje en sí.

Para el 2018, continuando con el aprendizaje de MC, la Universidad asumió un nuevo reto. Actualmente, cada 3 o 4 años maperos de distintas partes del mundo se reúnen para compartir sus experiencias de aprendizaje acerca del uso poderoso de los Mapas Conceptuales desarrollados por Joseph Novak en congresos internacionales. Sedes como Malta (2012), Brasil (2014), Estonia (2016), Beijing (2017) y Colombia (2018) han sido elegidas para recibir a la comunidad de maperos a compartir sus experiencias de aprendizaje. La última convocatoria se realizó en Medellín en septiembre del 2018 (el "Octavo Congreso Internacional sobre Mapas Conceptuales".

Ante dicha convocatoria, la Universidad Castro Carazo se propuso la elaboración de una ponencia que compartiera el transitar de la universidad, al sistematizar la experiencia de los maperos. El desafío consistía en elaborar un documento de carácter científico que representara la visión y experiencia de un equipo de diez autores. Con mucho entusiasmo, esta fue la única universidad de Costa Rica que presentó una ponencia internacional. Fue la titulada: "Transitar hacia un pensamiento de orden superior: mapas conceptuales en la Universidad Castro Carazo" (Badilla-Saxe et al., 2018).

La experiencia de preparar una ponencia internacional, abonó el terreno para una futura vicerrectoría de Investigación en la universidad, ya que la investigación es un ejercicio paralelo a la construcción de $\mathrm{MC}$ al permitir la negociación de significados y la identificación de concepciones equivocadas. Por otra parte, se demostró el proceso de aprendizaje transitado por la Comunidad de Maperos, así como la capacidad de trabajo colaborativo y transdisciplinario que ha traído con sí el PUEDO.

\subsubsection{Entornos virtuales de aprendizaje}

El modelo educativo de la universidad invita a transitar de la enseñanza hacia el aprendizaje. Es a partir de este modelo y sus fundamentos que se entiende el aprendizaje como un proceso continuo y ubicuo, que ocurre en todo momento y espacio; es decir, en multientornos. Los entornos virtuales de aprendizaje (EVA) se visualizan como un componente clave de los multientornos que facilita la universidad para acompañar el aprendizaje de toda la comunidad universitaria. Se trata de espacios atractivos, que 
aprovechan los medios digitales para acortar las distancias, acercando a docentes y estudiantes y a estudiantes entre sí, con el fin de favorecer la construcción conjunta de aprendizajes (Badilla-Saxe, 2015).

La Universidad, a través del A:Lab, provee a sus docentes de procesos de acompañamiento tecno-pedagógico (ATP), los cuales buscan una integración efectiva del modelo educativo en el diseño e implementación de los EVA que complementan la ejecución de los cursos presenciales que ofrece la universidad. A través de los ATP, se busca la adquisición progresiva de mayor confianza y fluidez en el uso de tecnologías digitales por parte del equipo docente, para garantizar EVAs atractivos, centrados en la persona y en la interacción. A diferencia de los repositorios digitales, los entornos virtuales de aprendizaje de la universidad facilitan la construcción conjunta de aprendizajes, e incentivan procesos de reflexión recursiva sobre lo que se aprende, cómo se aprende, y sobre las habilidades que se fomentan en los diferentes cursos y disciplinas (AdamsJohnson, Becker, Gago, García, y Martín, 2013; Badilla-Saxe, 2015; Morado y OcampoHernández, 2018).

\subsection{Innovación curricular}

Este proyecto estratégico busca permear en toda la oferta académica de la Universidad el modelo ecoformativo, además de crear nuevos productos académicos y de plasmar en las carreras, programas de especialización, técnicos y cursos libres los pilares del modelo. En cuanto a los programas académicos (PAC), se han hecho mejoras en todas las direcciones académicas tanto en contenidos, como criterios de evaluación y bibliografía, estos cambios requieren necesariamente de modificaciones en la metodología del aula, la cual se centra ahora en el aprendizaje y el aprendiente, siendo el PUEDO un pilar necesario para el traslado de los PAC a la realidad, es decir a la práctica docente, al preparar a los docentes para el cambio de visión. Mientras, para el 2020, la universidad está trabajando en la completa mejora de los cursos restantes de malla curricular, incluyendo un Doctorado en Educación.

Por otra parte, los nuevos técnicos, cursos libres y programas de especialización contemplan el aprendizaje en multientornos y un primer gran diferenciador: el Acuerdo de Aprendizaje, el cual contempla en primera instancia que el estudiantado esté de acuerdo con lo que aprenderá, el cómo y cuándo lo hará. El Acuerdo, a diferencia del PAC, le indica al aprendiz lo que aprenderá desde una visión crítica planteándole preguntas generadoras que fomentan la resolución de problemas y el análisis. Además, dicho acuerdo está redactado en función de lo que el estudiante deberá realizar en cada sesión. El segundo diferenciador está presente en todos los PAC que han sido mejorados durante este año, y es que se basan en una Evaluación auténtica del Aprendizaje (EAA), aspecto que también conforma parte de una de las dimensiones del PUEDO tituladas bajo el mismo nombre. La evaluación auténtica se define como:

una forma de valoración en la que los sujetos llevan a cabo actividades del "mundo real" para dar a conocer aplicaciones significativas de sus conocimientos y habilidades, ofreciendo [...] "evidencias" de su desempeño y su comprensión (Escobar-Londono, Castano, RuizRuiz, y Restrepo-Botero, 2016, 177). Se centra en el/la estudiante, quien deberá enfrentar situaciones complejas, sea de manera individual o grupal (Condemarín y Medina, 2000). Evidentemente, parte de contextos reales: escenarios lo más similares posible a los que se tienen en el ejercicio profesional

$$
\text { (Brown, 2015) }
$$

Es importante resaltar que tanto las mejoras realizadas a los PAC parte de los planes de estudios de las carreras, como los acuerdos de aprendizaje, se realizan con el personal 
docente y los directores académicos acompañados por el equipo de Innovación Curricular (IC). En las sesiones de trabajo se promueve el diálogo conjunto con la intención de reflexionar y no criticar entorno a las necesidades pedagógicas tanto del discente como del docente para que se propongan alternativas de acción en el aula (OcampoHernández, 2016).

\section{ANÁLISIS Y RESULTADOS}

\subsection{Sobre el PUEDO}

Como se menciona en el apartado de materiales y métodos, se realizó una consulta a 39 docentes del PUEDO que cursaron el módulo de "Didáctica Imprescindible" para conocer su percepción inicial sobre el rol docente.

Para ello se optó por realizar un análisis del discurso de las personas participantes para valorar sus impresiones iniciales ante la pregunta: “ ¿Cuál considero que debe ser el rol docente?", la cual contestaron mediante un vídeo individual. Este análisis consiste en verificar si el discurso brindado por las personas participantes refleja algún tipo de cambio en su manera de pensar y de acercarse a la docencia universitaria.

Sus primeras impresiones sobre esta pregunta se muestran en la Figura 2:

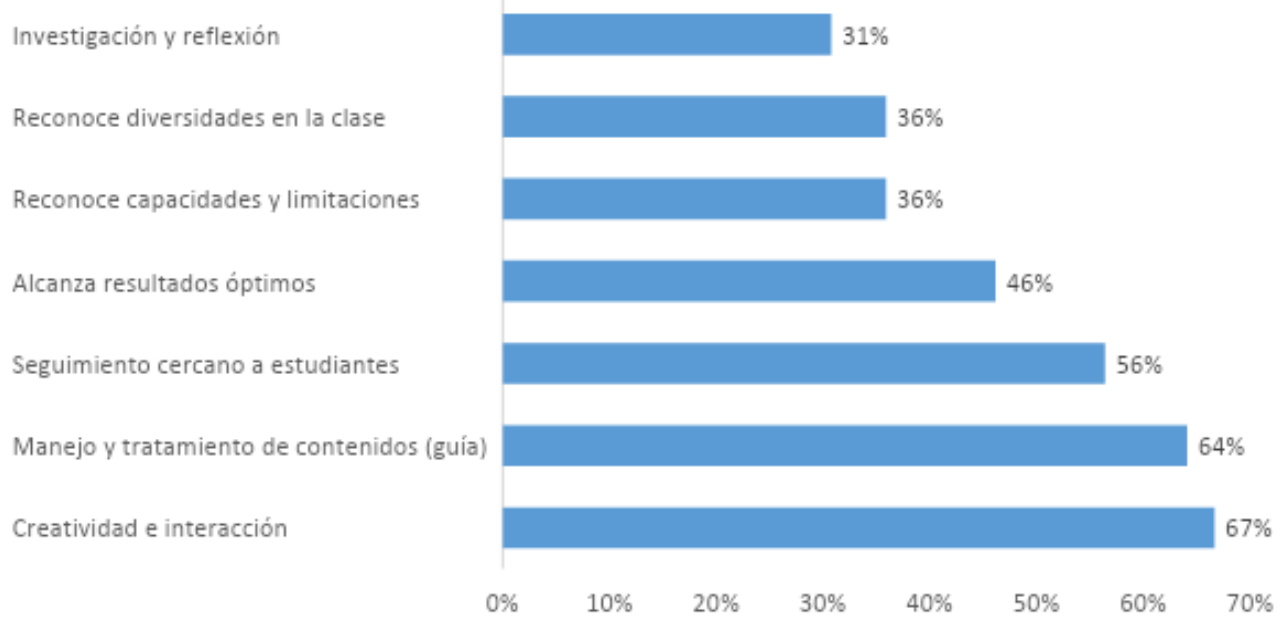

Figura 2. Primeras impresiones. Fuente: elaboración propia.

La imagen anterior muestra la agrupación de los discursos según su temática principal como respuesta a la pregunta formulada. Como se puede observar, más de la mitad del cuerpo docente consultado considera que su papel debe estar enfocado en promover espacios creativos e interactivos $(67 \%)$ y en el manejo y tratamiento de los contenidos (64\%), es decir, en la manera en la que se transmiten los conocimientos. Sin embargo, temas como la investigación o la reflexión no son tan mencionados por las personas participantes.

Si bien se da este énfasis en ser creativos y promover interacción en sus clases, las respuestas reflejan que, inicialmente, el cuerpo docente se encuentra en un proceso de "transición" del modelo "tradicional " de la educación, intentando hacer actividades innovadoras, pero se siguen mostrando ideas y prácticas tradicionales como el manejo y guía del docente como transmisor del conocimiento, dejando de lado el papel activo de las y los discentes en la construcción de su aprendizaje.

Luego de las primeras 5 semanas de capacitación, se consultó nuevamente a las mismas personas sobre cuál consideran que es el rol docente en los procesos de aprendizaje, y sus respuestas fueron las siguientes (ver Figura 3): 


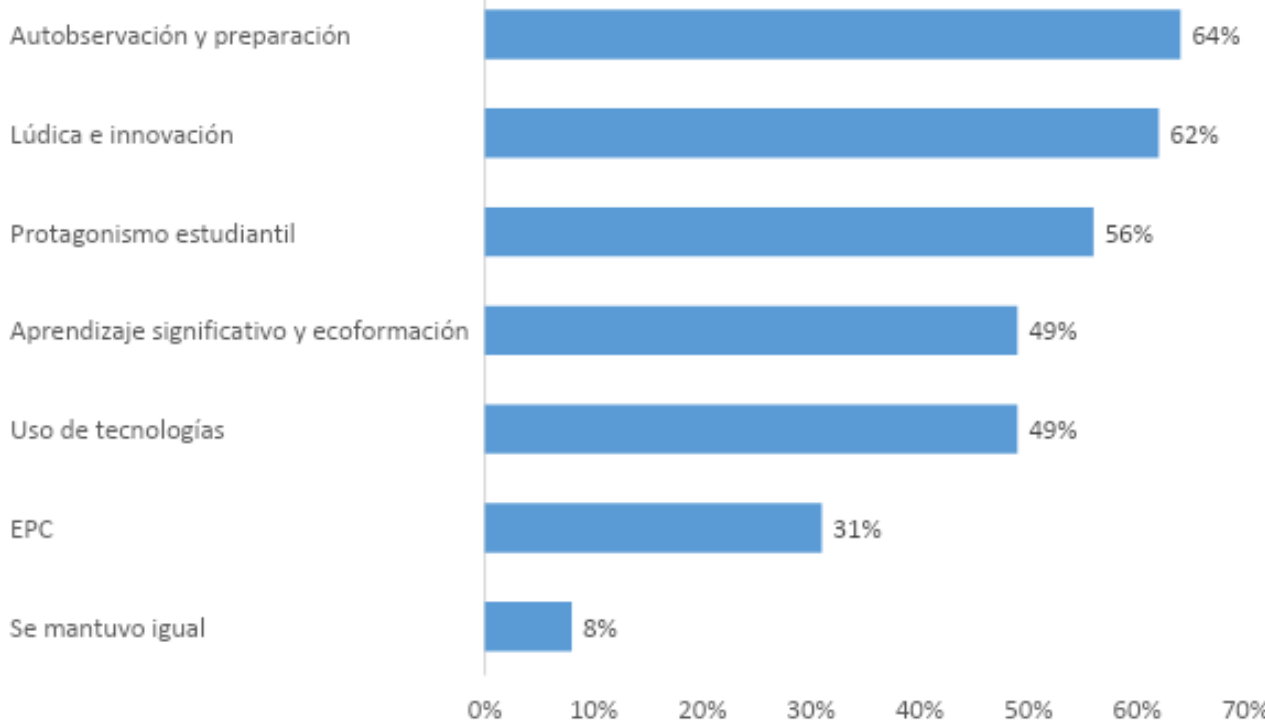

Figura 3. ¿Cómo cambió mi percepción del rol docente? Fuente: elaboración propia.

El modelo de ecoformación nos habla de la importancia de la interacción "yo - otros ambiente" y de reflexionar sobre la manera en que mis acciones tienen impacto en los demás. Esto se ve reflejado en las respuestas dadas por las personas consultadas, dado que un $64 \%$ mencionó como punto principal del rol docente la auto observación y la preparación, un tema que no estaba considerado inicialmente por las y los docentes.

Asimismo, más de la mitad (62\%) menciona la lúdica y la innovación como parte indispensable de los procesos de aprendizaje, de manera que las y los discentes cuenten con experiencias significativas y disruptivas que permitan un aprendizaje real. En este punto, abogan por el uso de TRICs (tecnologías de la relación, información y comunicación, Gabelas, Marta-Lazo, y Aranda (2012) como estrategias para generar esos espacios lúdicos tanto dentro como fuera de las clases y en la necesidad docente de innovar de manera continua.

De esta manera, fue vital para las y los docentes comprender que el uso educativo de las tecnologías va más allá de incorporar herramientas digitales en la clase, sino que estas herramientas deben responder a un modelo educativo disruptivo, que busca gestionar espacios colaborativos de discusión y construcción, y que permite una relación (incluso asincrónica y atemporal) con las demás personas, desarrollando comunidades de aprendizaje que aprenden unas de otras y que construyen en conjunto, en lugar de solo usar estrategias digitales en la clase para hacerla "más interesante".

En tercer lugar, se habla del protagonismo estudiantil (56\%), otro tema que no fue mencionado por las y los docentes en los comentarios iniciales, y que ahora ocupa relevancia, indicando que las personas participantes se encuentran más conscientes de que el proceso de aprendizaje se da de manera conjunta mediante la interacción discentes - docentes.

Estas respuestas permiten apreciar una mayor apropiación del modelo educativo por parte del cuerpo docente, quienes se sienten más empoderados para realizar estrategias lúdicas e innovadoras gracias al uso de tecnologías y los conocimientos adquiridos a lo largo del Programa de Especialización en Docencia Universitaria.

\subsection{Sobre el Laboratorio de Aprendizaje (A:Lab)}

Desde su inauguración, el A:Lab ha incubado y desarrollado más de 300 experiencias de aprendizaje a través de fabricación digital para estudiantes, docentes y comunidad 
educativa en general, impactando a un $40 \%$ de la población estudiantil activa de la universidad. En estas experiencias de aprendizaje se invita a imaginar, crear, fabricar y trabajar con otros y con otras disciplinas, participando de ambientes de aprendizaje innovadores con tecnología. Un ejemplo de estas fue el "Encuentro Internacional de Scratch", que se celebró en noviembre de 2017 con la presencia de Mitchel Resnick del Instituto Tecnológico de Massachusetts (MIT), creador del lenguaje Scratch y Marina Umaschi Bers de la Universidad Tufts y co-creadora de Scratch Jr.

Aunado a lo anterior, en cada período académico o cuatrimestre, se ha observado un aumento del $45 \%$ en la participación de docentes y estudiantes en las experiencias de fabricación digital, potenciando objetivos curriculares y desarrollando habilidades para la vida. El compromiso e involucramiento de la comunidad educativa también se ha demostrado por un aumento en la cantidad de docentes que transfieren lo aprendido en el A:Lab a sus prácticas dentro de aulas regulares; realizando una integración asertiva de las estrategias de aprendizaje activo y de los usos poderosos de las tecnologías digitales que se promueven en el A:Lab, para colaborar y construir aprendizajes desde cada disciplina.

Durante el 2019, la Universidad tuvo representación en un evento local TED en Costa Rica, denominado TEDxPuraVidaED, una edición dedicada a la educación. A través de la charla "Fabricando aprendizaje " se compartieron las lecciones aprendidas y buenas prácticas que ha ido acuñando la universidad para integrar la fabricación digital y el aprendizaje activo a nivel universitario (Aguilar, 2019).

En cuanto a los mapas conceptuales, quedan visibilizados con la ponencia en Colombia 2018: "Transitar hacia un pensamiento de orden superior: mapas conceptuales en la Universidad CastroCarazo " (Badilla-Saxe et al., 2018), la sistematización de un proceso de aprendizaje que se ha incubado desde el 2016 hasta el 2018 y que continúa impactando en la universidad con la implementación del PUEDO. En contraste, desde la primera generación de maperos hasta ahora, se cuentan con 68 maperos. Esto corresponde a personal docente y administrativo que iniciaron sus aprendizajes antes de existir el PUEDO. Adicionalmente, el total de docentes que han participado del programa y que han transitado la dimensión de Tecnología Disruptiva es de84 profesores y profesoras.

Además, el A:Lab ofrece talleres a los docentes con el fin de incorporar en sus dinámicas de clases los $\mathrm{MC}$. El número de estudiantes que han participado de dichos talleres en el último año es de 270 , de todas las carreras que ofrece la universidad. Lo anterior denota las líneas de acción por parte de la universidad al escoger el uso de CmapTools como herramienta para la representación gráfica del conocimiento.

Sobre el aprendizaje en multientornos, el A:Lab, ha permitido el acompañamiento tecnopedagógico de un $70 \%$ del cuerpo docente. Estos docentes reciben acompañamiento y asesoría personalizada, respetando sus tiempos de aprendizaje, para la generación de entornos virtuales congruentes con el modelo ecoformativo. Además, la implementación de estos ATP permitió el desarrollo de una investigación-acción para conocer, analizar y documentar la transformación vivida por la comunidad docente durante la transición de repositorios de documentos en línea a entornos virtuales de aprendizaje (Morado y Ocampo-Hernández, 2018). Actualmente, un 63\% de la oferta activa de cursos cuenta con un EVA. Esto representa un $70 \%$ de docentes que diseñan y utilizan al menos un EVA, siguiendo el nuevo modelo educativo de la universidad. Cada cuatrimestre, se ha percibido un aumento sostenido del $3 \%$ en la cobertura de entornos virtuales de aprendizaje.

\subsection{Sobre la Innovación Curricular}

En general los datos demuestran que, al estar conformados los planes de estudio por una malla curricular de al menos 30 cursos, se han realizado mejoras a más de la mitad de los PAC de la oferta académica. Siendo visible el esfuerzo de la universidad 
por ser coherente con aquellos documentos académicos que requieren mejoras por incluir al modelo ecoformativo. Únicamente las direcciones de Ingeniería Industrial y Matemáticas, Idiomas, muestran un número bajo de cursos mejorados, puesto que son direcciones académicas que ofrecen "cursos de servicio", por ejemplo: Cálculo, Matemáticas Discretas, inglés. En cuanto a Informática, se planea para este año 2020 una revisitación del $100 \%$ de los cursos de la carrera en Bachillerato.

Tabla 1. Lista de mejoras realizadas en Ios PAC por dirección académica a la luz del modelo ecoformativo 2019-2020. Fuente: elaboración propia.

\begin{tabular}{lll}
\hline $\begin{array}{l}\text { Direcciones } \\
\text { Académicas }\end{array}$ & Cursos mejorados & $\begin{array}{l}\text { Cursos a } \\
\text { mejorar en } \\
2020\end{array}$ \\
\hline $\begin{array}{l}\text { Dirección de } \\
\text { Aduanas y Negocios } \\
\text { Internacionales }\end{array}$ & 25 & 8 \\
$\begin{array}{l}\text { Dirección de } \\
\text { Administración de }\end{array}$ & 38 & 19 \\
Negocios & \\
$\begin{array}{l}\text { Dirección de } \\
\text { Contaduría }\end{array}$ & 14 & 1 \\
$\begin{array}{l}\text { Dirección de Derecho } \\
\text { Dirección de Ing. }\end{array}$ & 24 & 7 \\
Industrial y & 5 & 3 \\
Matemáticas & & 0 \\
Dirección de Idiomas & 5 & 17 \\
$\begin{array}{l}\text { Dirección de } \\
\text { Informática }\end{array}$ & 2 & 70 \\
Totales & 127 & \\
\hline
\end{tabular}

\section{DISCUSIÓN Y CONCLUSIONES}

Con el compromiso para llevar a la práctica el modelo ecoformativo han surgido diversidad de buenas prácticas educativas en la Universidad Castro Carazo, las cuales han impactado al alumnado, docentes, personal administrativo e, incluso, en la infraestructura y mobiliario de la universidad. Estas prácticas se han desarrollado de manera articulada y siempre en congruencia con el modelo educativo, en miras de construir una cultura organizacional que promueva la innovación en todos los ámbitos de acción de este centro educativo.

La universidad ha comprendido que dicha innovación no ocurre de manera lineal, ni por implementaciones jerárquicas tradicionales "de arriba a abajo". En cambio, la innovación crece desde adentro, "de abajo hacia arriba", e implica trabajar con toda la comunidad universitaria (facultades, estudiantado y administrativos) en pasos pequeños pero constantes. Además, entiende la tecnología como un fenómeno social, por lo que el interés principal es encontrar los usos relevantes y creativos que puedan mejorar los resultados de aprendizaje esperados.

Esta visión holística comprende una serie de acciones y supuestos clave para la visibilización del modelo ecoformativo:

- La educación estimula más a separar que a relacionar, desde el modelo ecoformativo Castro Carazo, se busca que desde cada proyecto seamos capaces de relacionarnos y de reconocer la interdependencia generalizada de todo y de todos hacia la ruta de aprendizaje por visibilizar el modelo educativo en los discursos y prácticas educativas. 
- Es importante involucrar a toda la comunidad educativa (especialmente las y los docentes) en el proceso de transición hacia el cambio de modelo, de manera que, no solo dominen los conceptos del modelo como tal, sino que cuenten con herramientas que les permitan una reflexión permanente sobre su quehacer docente. En este sentido, el cambio en el discurso reflejado a través de las respuestas de las y los profesores refleja una concientización sobre la importancia de problematizar y analizar sus propias estrategias docentes.

- Transicionar de la visión de TICs a TRICs implica ver las tecnologías no solo como una fuente de información y comunicación, sino entender el potencial de las tecnologías, relacionarnos, creando y fomentando comunidades de aprendizaje continuas, que desarrollen una espiral virtuosa y que permitan la realimentación y la ecología de saberes: compartimos experiencias y conocimientos, construimos en conjunto y reconstruimos a partir de los esfuerzos de las demás personas (Gabelas et al., 2012). Al final, todos los procesos están conectados y cada acción (individual y colectiva) es parte del proceso de aprendizaje.

- Estas prácticas y procesos de (re) construcción continua y diálogo permanente entre las diversas personas de la comunidad educativa, permiten valorar nuevas formas de entender y vivir la calidad académica universitaria, al considerar estos espacios como indicadores de procesos de autoevaluación continua. En este sentido, el uso de Tecnologías de la Relación, la Información y la Comunicación (TRICs) es de vital importancia para generar esos espacios de discusión y mejora continua (Figueroa-Céspedes, 2016).

\section{REFERENCIAS}

Acuña, M. (2017). Guía hacia una Evaluación Auténtica en la Universidad Castro Carazo. San José, Costa Rica: Universidad Castro Carazo.

Adamini, M. (2016). Aproximaciones al análisis del discurso en los estudios identitarios.

Relmecs. Revista Latinoamericana de Metodología de las Ciencias Sociales, 6(1), 1-20. Descargado de

http://www.relmecs.fahce.unlp.edu.ar/article/view/relmecs_v06n01a06/7277

Adams-Johnson, L., Becker, S., Gago, D., García, E., y Martín, S. (2013). NMC Perspectivas Tecnológicas: Educación Superior en América Latina 2013- 2018. Un Análisis Regional del Informe Horizon del NMC.

Aguilar, V. (2019). Fabricando Aprendizaje. Descargado de https://www.youtube.com/watch?v=ElsXEhmEfdQ

Badilla-Saxe, E. (2005). Educación, relaciones y conexiones . Ágora(1).

Badilla-Saxe, E. (2015). Educación sin distancia. Descargado de https://www.nacion.com/opinion/foros/eleonora-badilla-educacion-sin-distancia/ Z3KMRFCALJDERAGDCWU35K5T7Y/story/

Badilla-Saxe, E. (2018a). La crisis planetaria y la ecoformación. Descargado de https://delfino.cr/2018/02/la-crisis-planetaria-la-ecoformacion

Badilla-Saxe, E. (2018b). Mapas Conceptuales (Video). Descargado de https://youtu.be/bJ_6X_rT6HU

Badilla-Saxe, E., Ocampo-Hernández, S., Acuña-Picado, S., Carvajal-Rivera, M., Vargas-González, I., Cordero-Arroyo, J., ... Salazar-Sáenz, M. (2018). Transitar hacia un pensamiento de orden superior: mapas conceptuales en la Universidad Castro Carazo . Concept Mapping Conference: Renewing Learning and Thinking. Proceedings of the 8th International Conference on Concept Mapping. Descargado de https://cmc.ihmc.us/cmc2018Proceedings/CMC2018\%20Proceedings.pdf.zip

Barnechea-García, M., y Morgan-Tirado, M. (2010). La sistematización de experiencias: producción de conocimientos desde y para la práctica. Tend. Retos(15), 97-107. 
Descargado de http://www.ts.ucr.ac.cr/binarios/tendencias/rev-co-tendencias-15-07.pdf

Brown, S. (2015). La evaluación auténtica: el uso de la evaluación para ayudar a los estudiantes a aprender. RELIEVE. Revista Electrónica de Investigación y Evaluación Educativa, 21(2), 1-10. https://doi.org/10.7203/relieve.21.2.7674

Cañas, A. J., Reiska, P., y Möllits, A. (2017). Developing higher-order thinking skills with concept mapping: A case of pedagogic frailty. Knowledge Management \& E-Learning: An International fournal, 9(3), 348-365. https://doi.org/10.34105/j.kmel.2017.09.021

Castro, S. (2009). Piaget, Chomsky y neurociencia. Descargado de http://wvw.nacion.com/ln_ee/2009/febrero/08/opinion1868010.html

Collazos, C., Guerrero, L., y Vergara, A. (2002). Aprendizaje colaborativo: Un cambio en el rol del profesor. Descargado de http://users.dcc.uchile.cl/ luguerre/papers/CESC-01.pdf

Condemarín, M., y Medina, A. (2000). Evaluación auténtica de los aprendizajes: un medio para mejorar las competencias en lenguajes y comunicación. Santiago, Chile: Andrés Bello.

Escobar-Londono, J. V., Castano, D. A., Ruiz-Ruiz, M. P., y Restrepo-Botero, J. C. (2016). Evaluación auténtica del impacto social de procesos, proyectos y productos de investigación universitaria: un acercamiento desde los grupos de investigación. Revista Lasallista de Investigación, 13(1), 166-180. Descargado de http://www.scielo.org.co/pdf/rlsi/v13n1/v13n1a14.pdf

Figueroa-Céspedes, I. (2016). Rol mediador de aprendizajes en Educación Parvularia: procesos de apropiación en el contexto de una propuesta formativa en experiencia de aprendizaje mediado. Summa Psicológica, 13(1), 1-287.

https://doi.org/10.18774/summa-vol13.num1-287

Freire, P. (2002). Pedagogía de la Esperanza. Buenos Aires, Argentina: Siglo XXI Editores.

Gabelas, J. A., Marta-Lazo, C., y Aranda, D. (2012). Por qué las TRIC y no las TIC. COMeIN. Revista de los Estudios de Ciencias de la Información y de la Comunicación, 9, 2014-2226. Descargado de https://www.uoc.edu/divulgacio/comein/es/numero09/articles/ Article-Dani-Aranda.html

Henao-Willis, M. (1999). La Educación del futuro: Edgar Morin, un escenario global, Gabriel García Márquez un escenario local. Colombia. Ciencia y Tecnología de Colciencias, 17(4).

Jara, O. H. (1994). Para Sistematizar Experiencias. San José, Costa Rica: Publicaciones Alforja. Magisterio. (2008). Editorial (Vol. 33) (no 6). Descargado de http://cepalforja.org/sistem/documentos/revista_magisterio_33.pdf

Morado, M. F., y Ocampo-Hernández, S. (2018). Una experiencia de acompañamiento tecno-pedagógico para la construcción de entornos virtuales de aprendizaje en educación superior. Educación. Revista de la Universidad de Costa Rica, 43(1), 43-60. https://doi.org/10.15517/revedu.v43i1.28457

Moraes, M. C. (2010). Transdisciplinariedad y Educación (Vol. 6). Instituto Paulo Freire de España. Descargado de http://www.rizoma-freireano.org/index.php/ transdisciplinariedad-y-educacion--maria-candida-moraes

Novak, J. (2010). Learning, Creating, and Using Knowledge: Concept maps as facilitative tools in schools and corporations. Journal of e-Learning and Knowledge Society, 6(3), 21-30. Descargado de http://rodallrich.com/advphysiology/ausubel.pdf

Novak, J. D., y Gowin, D. B. (1984). Learning How to Learn. New York: Cambridge University Press.

Ocampo-Hernández, S. (2016). Supporting Educational Needs and Interests of Teachers from the Accompaniment and Listening of The Researcher-teacher. Revista Electrónica Educare, 20(2), 1-19. http://dx.doi.org/10.15359/ree.20-2.20

Papert, S. (1990). MIT Media Lab Epistemology and Learning Memo No. 2. Descargado de http://www.papert.org/articles/ACritiqueofTechnocentrism.html

Picado-Alfaro, M. (2018). Didactical Suggestions to Implement a Leveling Course in Mathematics: Systematization of an Experience at the National University of Costa Rica. Educare, 22(3), 314-331. http://dx.doi.org/10.15359/ree.22-3.15 
Salas-Alvarado, J. (2016). El aprendizaje colaborativo en la clase de Inglés: El caso del curso de Comunicación Oral I de la Sede del Pacífico de la Universidad de Costa Rica. Educación: revista de la Universidad de Costa Rica, 40(2), 1-19.

https://doi.org/10.15517/REVEDU.V40I2.16285 